\title{
Value of multidetector computed tomography image segmentation for preoperative planning in general surgery
}

\author{
Vincenzo Ferrari • Marina Carbone • \\ Carla Cappelli $\cdot$ Luigi Boni · Franca Melfi • \\ Mauro Ferrari · Franco Mosca · Andrea Pietrabissa
}

Received: 26 January 2011/ Accepted: 22 August 2011/Published online: 23 September 2011

(C) The Author(s) 2011. This article is published with open access at Springerlink.com

\begin{abstract}
Background Using practical examples, this report aims to highlight the clinical value of patient-specific three-dimensional (3D) models, obtained segmenting multidetector computed tomography (MDCT) images, for preoperative planning in general surgery.

Methods In this study, segmentation and 3D model generation were performed using a semiautomatic tool developed in the authors' laboratory. Their segmentation procedure is based on the neighborhood connected regiongrowing algorithm that, appropriately parameterized for the
\end{abstract}

V. Ferrari $(\varangle) \cdot$ M. Carbone $\cdot$ M. Ferrari · F. Mosca .

A. Pietrabissa

EndoCAS Center, Università di Pisa, Edificio 102, Ospedale di

Cisanello, Via Paradisa 2, 56124 Pisa, Italy

e-mail: vincenzo.ferrari@endocas.org

M. Carbone

Scuola Superiore Sant'Anna, Pisa, Italy

C. Cappelli

Divisione di Radiologia Diagnostica e Interventistica,

Dipartimento di Oncologia dei Trapianti e delle Nuove

Tecnologie in Medicina, Università di Pisa, Pisa, Italy

L. Boni

Minimally Invasive Surgery Research Center, Dipartimento di

Scienze Chirurgiche, Università dell'Insubria, Varese, Italy

F. Melfi

Dipartimento Cardio Toracico e Vascolare, Università di Pisa, Pisa, Italy

A. Pietrabissa

Fondazione IRCCS Policlinico San Matteo Pavia,

Università di Pavia, Pavia, Italy anatomy of interest and combined with the optimal segmentation sequence, generates good-quality 3D images coupled with facility of use. Using a touch screen monitor, manual refining can be added to segment structures unsuitable for automatic reconstruction. Three-dimensional models of 10 candidates for major general surgery procedures were presented to the operating surgeons for evaluation. A questionnaire then was administered after surgery to assess the perceived added value of the new technology. Results The questionnaire results were very positive. The authors recorded the diffuse opinion that planning the procedure using a segmented data set allows the surgeon to plan critical interventions with better awareness of the specific patient anatomy and consequently facilitates choosing the best surgical approach.

Conclusions The benefit shown in this report supports a wider use of segmentation software in clinical practice, even taking into account the extra time and effort required to learn and use these systems.

Keywords General surgery - Image segmentation · MDCT · Patient-specific 3D models · Surgical planning · Technology

Advancements in computed tomography (CT) and magnetic resonance imaging (MRI) scanners together with the introduction of new contrast mediums allow the acquisition of volumetric data sets that can describe the human anatomy and organ functionality as well as their diseases with highest level of detail. Medical data sets usually are composed of many phases, each comprising hundreds of images. Radiologists usually build a "mental model" of the specific anatomy, in general examining the volumetric data set slice by slice. The reading of medical data sets is 
therefore quite difficult, requiring specific knowledge and a large experience [1]. This entails information on how the contrast medium flows inside the anatomic structures and geometric skills for "mentally" visualizing the threedimensional (3D) development of the target anatomy, starting from sequences of 2D images.

The detailed information contained in a volumetric data set is fully used during the diagnostic phase but can be partially lost as it passes from the radiologic department to the surgical department. In fact, it is common practice for surgeons to plan the intervention using only the limited information provided by the radiologist, which consists of the textual diagnosis coupled with a few significant 2D images selected from the volumetric data set and occasionally with some static 3D reconstructions, again generated and chosen by the radiologist.

Three-dimensional reconstruction tools generally are included in most commercial radiologic software suites [2]. For example, maximum intensity projections (MIP) are commonly used in diagnosis [3-5]. An MIP comprises the $2 \mathrm{D}$ projection of a portion of the acquired volume, given the position of the projection plane and the volume limits, to represent much $3 \mathrm{D}$ information in a single $2 \mathrm{D}$ image. It is useful especially for representing the vascularization in a limited volume. In the case of large volumes, the information will be lost due to the superimposition of the various structures. For these cases, the user can visualize the scene using direct volume-rendering tools that provide a complete and simple 3D visualization [6-8]. These tools require that each intensity value of the data set be mapped to color and opacity values. The user can select standard transfer functions, or the user can define a particular function using ramps, piecewise linear functions, or arbitrary tables.

Standard transfer function windows generally are predesigned for the visualization of various anatomic structures. However, in some particular cases, direct volume rendering does not allow correct representation of the 3D information. For example, with a CT angiography of an abdominal aortic aneurysm, because the blood flows inside the parietal thrombus, the latter is not enhanced by the contrast medium and therefore is not visible using the standard transfer functions for arteries. An attempt to include in the window the values of the thrombus, including many other voxels with the same intensity of signal, will be difficult, and the resulting scene will be somehow confused.

Both MIP and direct volume rendering are based on the intensity and not the nature of the voxels. They provide a fast and easy 3D visualization but with considerable drawbacks. For a better rendering of complex anatomic regions, more advanced tools and longer working times usually are required.
The theoretical advantages offered by generating 3D models through segmentation are well known $[9,10]$. Segmentation, which characterizes each voxel by its nature and not only by its intensity, allows better visualization and consequently facilitates understanding of the anatomy. This is exemplified by the ability to turn models of the various organs in the scene on and off and the ability to regulate their transparency. In addition, it makes possible functions typical of simulation, allowing interaction with the anatomy, thanks to the geometric characterization of structures [11-13].

Segmentation tools can be categorized on the basis of their automation level. Completely automatic segmentation software is inapplicable in the abdominal area due to the presence of many anatomic variations, particularly regarding vascularization. Semiautomatic segmentation software requires the interaction of the user to set parameters such as seed points or thresholds and to verify and eventually repeat the procedure with a different setting in function of the result. Finally, manual segmentation tools allow the user to perform segmentation that colors the images using pen-like instruments or contours. Manual segmentation is slow but can be useful after a semiautomatic segmentation to refine the borders of the anatomic structures on the basis of the anatomic knowledge and the capacity to interpret the images of the user. In this case, semiautomatic segmentation software allows the segmentation work to be speeded up, but the quality of the result is driven by the user, a radiologist.

Currently, the market and the open source community have made segmentation tools available that sometimes are integrated with the radiologic software (e.g. Analyze by Mayo Research, Scottsdale, USA; Amira by Visage Imaging, Richmond, Australia; Osirix by University Hospital of Geneva, Switzerland).

Nonetheless, segmentation is not widely used in clinical practice, probably because of the time required to learn and use the software. At the Center for Computer-Assisted Surgery of the University of Pisa "EndoCAS" (www. endocas.org), we have developed an integrated software to generate patient-specific 3D models of the target anatomy for use in the preoperative planning process. This article evaluates the potential of multidetector computed tomography (MDCT) image segmentation for preoperative planning in general surgery for a short series of patients undergoing major surgery. The segmentation was performed using semiautomatic segmentation software developed in our laboratory that allows patient-specific 3D models of abdominal organs to be obtained quickly and easily. The described methodology can be performed using other manual or semiautomatic tools to obtain the same results in terms of information for the surgical planning, but this will require spending more time and having the 
knowledge to deal with a complex algorithm not easy for nontechnical users to apply.

\section{Materials and methods}

The described methodology was in use at the EndoCAS Center for computer assisted surgery based at Cisanello Hospital, Pisa between January 2009 and May 2010. In every case, MDCT images were segmented after their anonimization and stored in separated directories organized by patient number and phase identifier. All the data sets contain a basal phase (slice thickness, 1.25-5 mm), an arterial phase (slice thickness, 0.6-2.5 mm), and a venous phase (slice thickness, 0.6-2.5 mm). Some data sets contain a pancreatic/portal phase (slice thickness, 0.6-1.25 $\mathrm{mm}$ ).

Patient-specific 3D models were generated using a semiautomatic tool [14] that we integrated into the open source software ITK-SNAP 1.5 (www.itksnap.org) [15]. The whole segmentation procedure is based on the neighborhood connected region growing algorithm appropriately parameterized for the specific anatomy. The segmentation process is performed by the radiologist in collaboration with the operating surgeon, who clarifies his or her needs in view of the proposed surgical procedure.

The basic algorithm integrated in the "EndoCAS segmentation pipeline" is guided by the radiologist in the process of generating 3D image reconstruction (semiautomatic). During segmentation, the topology of segmented structures is validated "online" by the radiologist and corrected if necessary, steering the appropriate parameters.

The system allows generation of the 3D abdominal models used to plan most general surgery procedures in about $30 \mathrm{~min}$. Manual refining through a touch screen monitor is used occasionally. This is particularly useful for the segmentation of structures impossible to identify in a automatic/semiautomatic manner. In these circumstances, after the semiautomatic segmentation procedure, we start the manual total segmentation, or in most cases, just a refinement of the structures that require a perfect geometric description of their borders for the safe performance of the intervention. We can use different types of manual segmentation tools such as a pen to plot points (with different sizes and shapes and with the possibility of plotting 3D points) or contours (automatically filled). Inside the segmentation software, it also is possible to simulate cutting planes and perform a volume estimation of each separated segment. This is particularly useful in hepatic surgery.

The MDTC data set of 10 consecutive patients about to undergo major surgery was used to generate 12 3D models of the target anatomies. For two patients with liver metastasis, the intervention was evaluated twice: after the intervention and before embolization of the portal vein.
The operating surgeon was involved in the generation process, as described earlier. After the surgery, he or she was asked to rate the perceived benefit of the new technology compared with the conventional CT images by answering six items of a specifically designed questionnaire (Table 1).

Grading of perceived improvement involved choosing a value between 1 (no value) and 5 (very high value). The following areas were investigated: visualization of the target anatomy, relationships with neighboring structures, value for the preoperative planning, value for teaching purposes (e.g., medical students, residents), value for intraoperative use (orientation, navigation), and effect in changing the surgical strategy.

\section{Results}

Case 1: tumor at the main bifurcation of the biliary tree (Klatskin tumor)

The regions segmented were the primary tumor, liver parenchyma, gallbladder, biliar ducts, hepatic arteries, and portal and hepatic veins. Manual refinement was required for the biliar tree. The 3D model shown in Fig. 1B and C was used for preoperative planning. It shows the exact relationship between the lesion and the surrounding structures and allowed the surgeon to simulate the resection plane, optimizing the intervention, a right hepatectomy. The visual information was made available to the entire surgical team through pre- and intraoperative display of the 3D models.

\section{Case 2: liver metastases 1}

The patient in this case had two metastases from colorectal cancer in the right hepatic lobe. An adequate healthy residual liver volume is mandatory for the performance of a safe right hepatectomy to provide sufficient postoperative hepatic function. After segmentation (almost all of it semiautomatic except for the vena cava), the surgeon simulated the resection plane. A residual hepatic volume of $323 \mathrm{~cm}^{3}$ (Fig. 2A), as estimated by the software, was deemed insufficient for the body mass index of this patient. A right portal vein embolization then was percutaneously performed, which is a common approach in similar cases. After 6 weeks, the patient's anatomy was again acquired with the MDCT scanner. After segmentation and hepatectomy simulation, our evaluation showed that the left lobe of the liver had increased to $467 \mathrm{~cm}^{3}$ (45\% of growth, Fig. 2B), and the surgeon proceeded safely to the intervention. 
Table 1 Opinion of each surgeon regarding the level of improvement offered by the three-dimensional (3D) models Vs the standard radiologic images commonly used for preoperative planning

The levels of improvement are indicated as 1 (none), 2 (marginal), 3 (significant), 4 (good), or 5 (very good)

\begin{tabular}{|c|c|c|c|c|c|c|c|c|c|c|c|}
\hline \multirow[t]{2}{*}{ Area } & \multicolumn{10}{|c|}{ Patient } & \multirow{2}{*}{$\begin{array}{l}\text { Overall mean } \\
\text { value }\end{array}$} \\
\hline & 1 & 2 & 3 & 4 & 5 & 6 & 7 & 8 & 9 & 10 & \\
\hline $\begin{array}{l}\text { Visualization of the target } \\
\text { anatomy }\end{array}$ & 5 & 5 & 5 & 5 & 5 & 5 & 5 & 5 & 5 & 5 & 5.0 \\
\hline $\begin{array}{l}\text { Relations with neighboring } \\
\text { structures }\end{array}$ & 5 & 5 & 4 & 5 & 4 & 2 & 3 & 5 & 5 & 2 & 4.0 \\
\hline Value for preoperative planning & 4 & 5 & 5 & 4 & 3 & 3 & 5 & 4 & 5 & 2 & 4.0 \\
\hline Value for teaching purposes & 5 & 5 & 5 & 5 & 2 & 5 & 3 & 5 & 5 & 4 & 4.4 \\
\hline Value for intraoperative use & 4 & 2 & 2 & 2 & 5 & 3 & 2 & 5 & 5 & 2 & 3.2 \\
\hline $\begin{array}{l}\text { Effect in changing the surgical } \\
\text { strategy }\end{array}$ & 2 & 5 & 5 & 3 & 4 & 2 & 5 & 4 & 4 & 2 & 3.6 \\
\hline Overall mean value & 4.2 & 4.5 & 4.3 & 4.0 & 3.8 & 3.3 & 3.8 & 4.7 & 4.8 & 2.8 & \\
\hline
\end{tabular}

Fig. 1 A Computed tomography (CT) slice with superimposed segmentation. Note the tumor positioning. B, C Three-dimensional (3D) rendering of the segmented data set. Structures can be removed or made transparent for exact visualization of tumor positioning and tumor relationship with vessels and biliar ducts

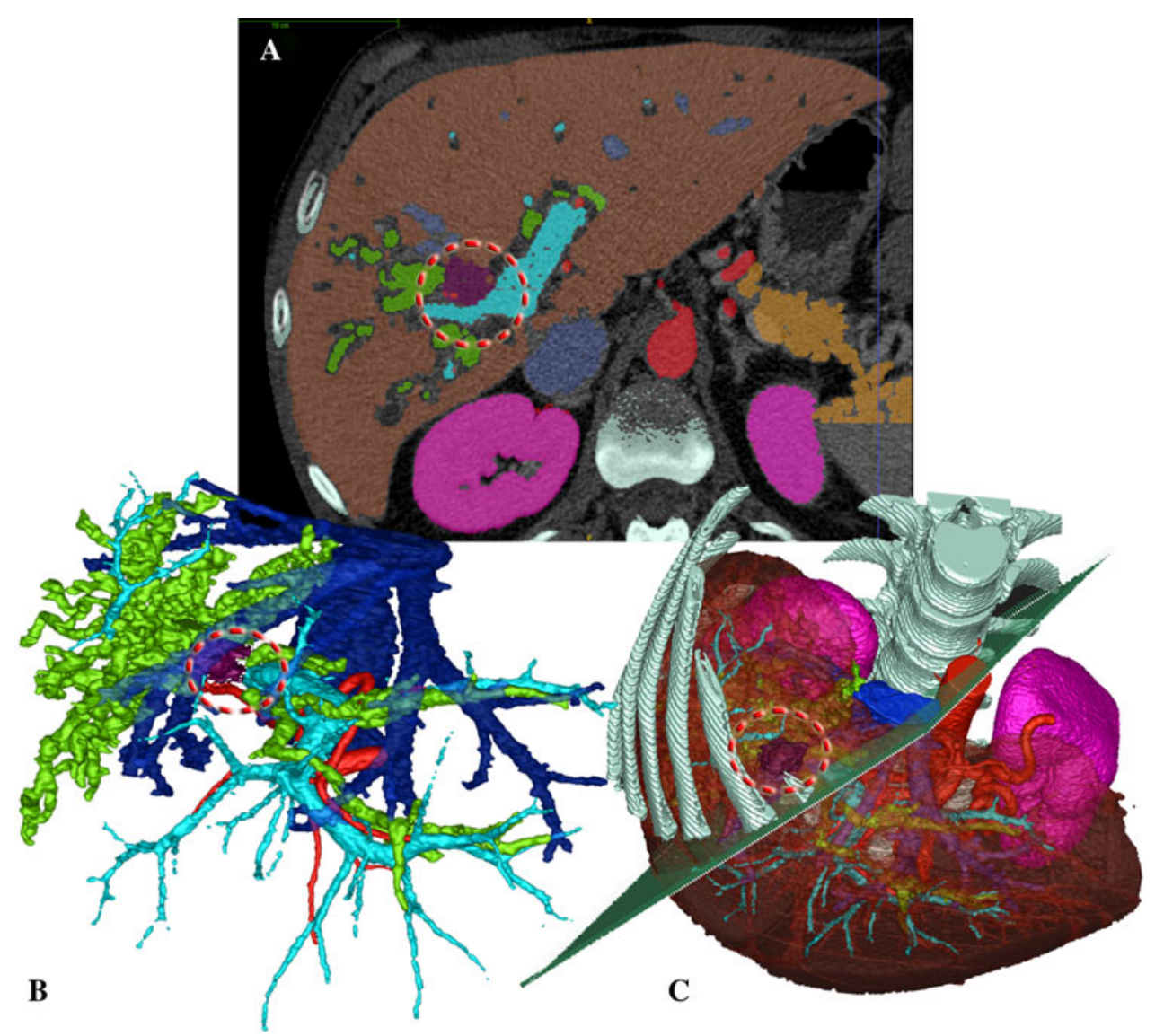

Case 3: liver metastases 2

As with the previous case, we built a 3D model and verified that the residual part of the liver after the proposed hepatectomy was not sufficient. Again, the surgeon decided to perform a percutaneous embolization of the right portal system. After 6 weeks, the standard CT diagnosis described "about $20 \mathrm{~mm}$ of expansion in the transverse diameter of the left lobe," with no information about volume change. The volume computed on the 3D model established an inadequate hypertrophy of the left lobe. This was increased only from 230 to $310 \mathrm{~cm}^{3}$ (Fig. 2C). The proposed hepatectomy was aborted.

Case 4: primary liver tumor

The liver was segmented and reconstructed. The surgeon simulated various resection planes to identify the best 
Fig. 2 A First segmented model showing liver vessels, metastases, and parenchyma. The residual healthy volume is estimated. B Three-dimensional (3D) model 6 weeks after embolization of the right portal system. Vasculature metastasis, embolized vessels, and parenchyma are rendered. The residual healthy volume is $45 \%$ larger. C A similar case after embolization in which the embolization did not achieve the hoped hypertrophy of the left lobe
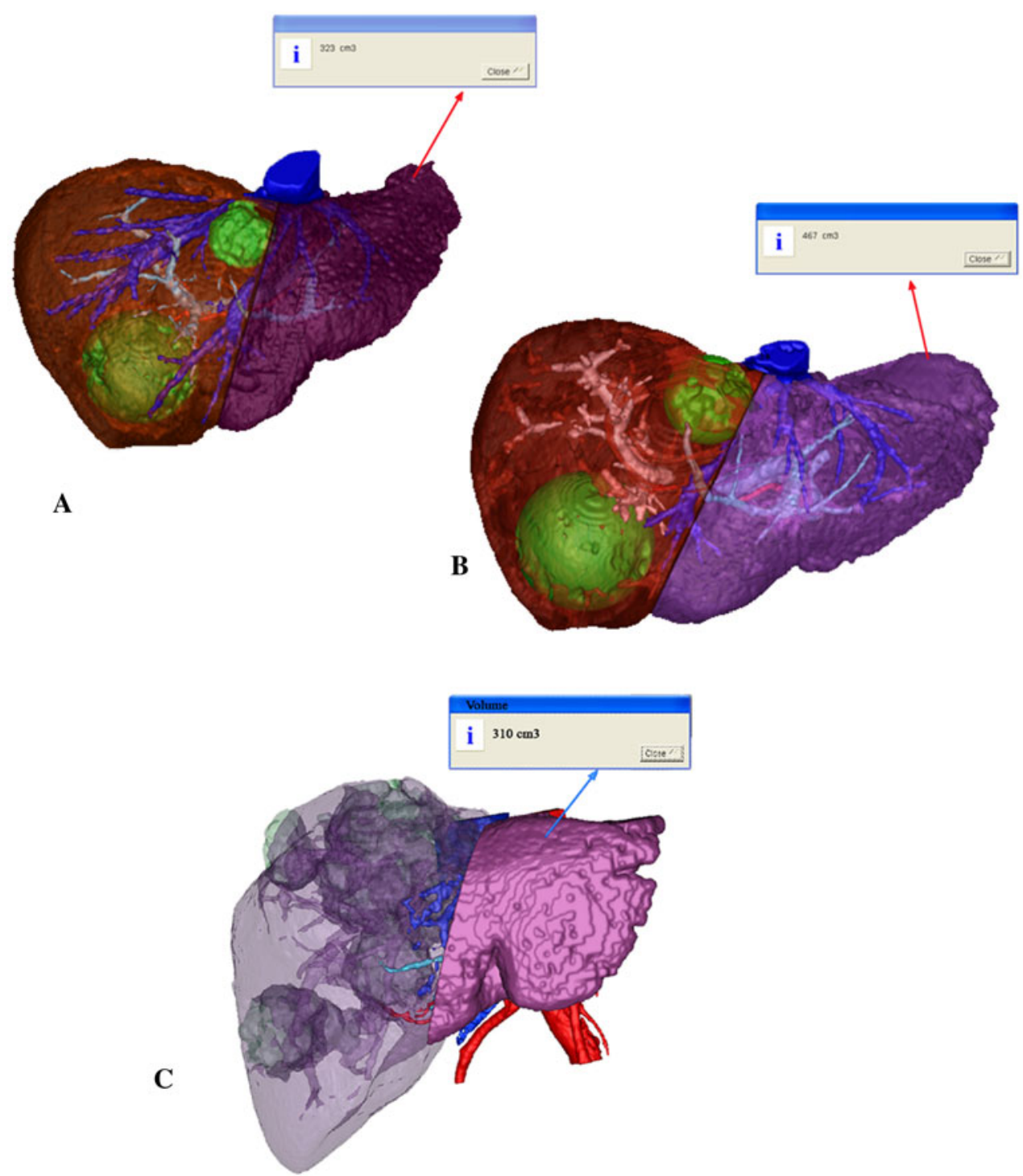

intraoperative strategy and to optimize the residual hepatic volume. The obtained 3D model showed that the lesion involved most of the cranial hepatic segments but not the main vessels of the area (major hepatic veins) (Fig. 3). The surgeon thought that enucleation of the lesion was feasible, with avoidance of a more invasive regulated hepatectomy (hypothesized watching only the CT diagnosis).

\section{Case 5: pancreatic tumor}

The 3D reconstruction (Fig. 4) generated for this case involving a neuroendocrine lesion of the pancreas was used by the surgeon to understand the relationship between the tumor and the surrounding structures to determine the surgical strategy: a laparoscopic distal pancreatectomy. The 3D model also was used in the operating room as input data for the EndoCAS Navigator [16] to achieve intraoperative identification of the tumor.
Case 6: lung tumor

A major lung resection was performed for a 50-year-old smoker with non-small cell lung cancer. After model generation (done entirely with semiautomatic segmentation), the surgeon decided to proceed with a robotic right lower lobectomy. The reconstruction showed the exact position of the lesion with respect to the surrounding vessels and bronchus (Fig. 5). The 3D visualization of the lung anatomy allowed the surgeon to preview the anatomic distribution of the vessels and the bronchial tree to plan the operation. Video robotic lobectomy follows the standard surgical steps of open thoracic surgery. The surgical sequence implies division of the artery as a first step, followed by division of the vein and finally of the bronchus. In this case, the $3 \mathrm{D}$ visualization was not considered useful for understanding the relationships with neighboring structures and for changing the surgical strategy because 

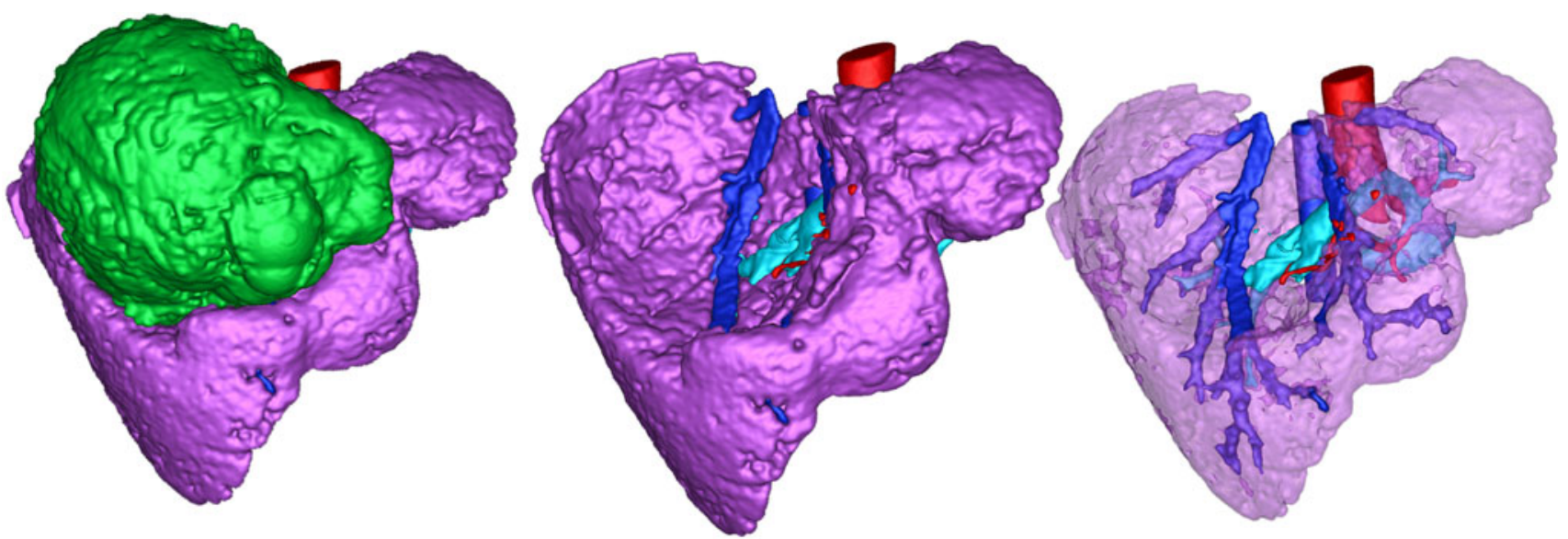

Fig. 3 Large epatic lesion that involves most of the upper liver segments. Beyond the tumor, it can be seen that the vascular system is only marginally involved and that the tumor can be safely enucleated

Fig. 4 Three-dimensional (3D) model showing the little lesion in the pancreas parenchyma, the path of the vessels, and their positions

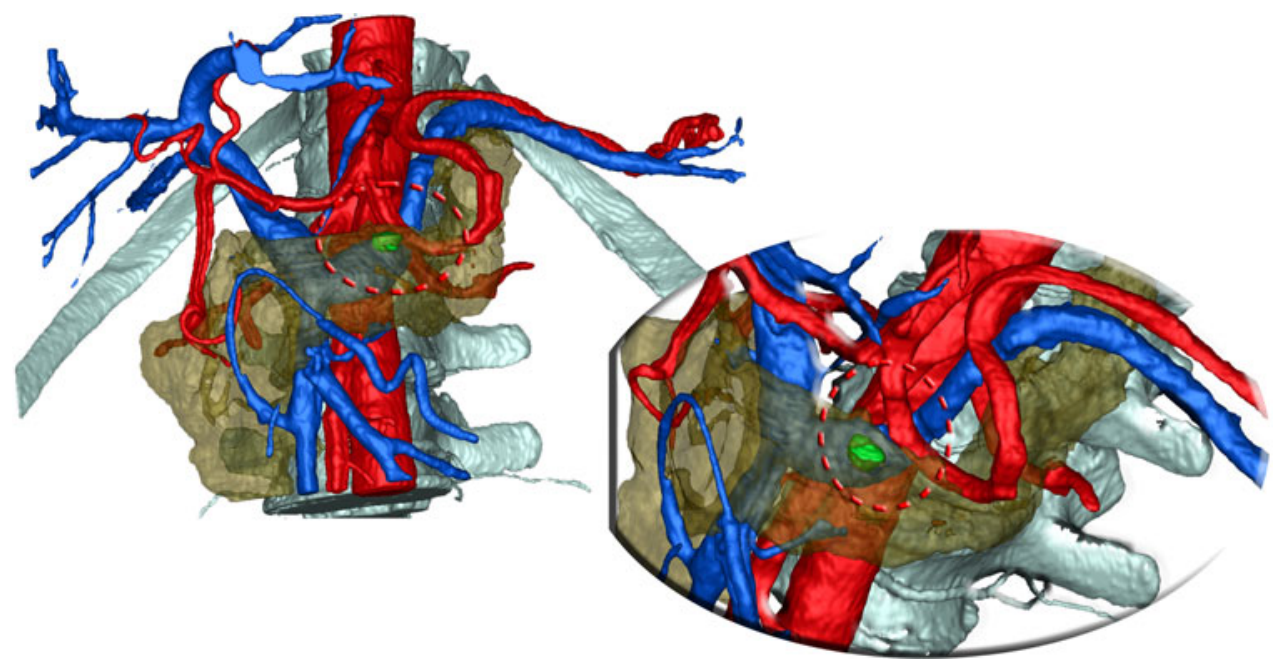

lobectomy is an intervention with complete removal of a lung segment and thus it is not necessary to proceed to a selective removal of the vessels around the tumor.

Case 7: aneurysm of the hepatic artery

In this case involving an aneurysm of the common hepatic artery, direct volume rendering did not allow precise definition of its origin in terms of afferent and efferent vessels (Fig. 6). Based only on this type of visualization, the surgeon would intervene with open surgery because the laparoscopic approach is deemed too difficult, and endovascular repair would require positioning of a stent in a segment of artery with too many collaterals. Starting with the rough model obtained using the semiautomatic algorithm, we manually refined the aneurysm morphology using the touch screen. We started with slices that had sharper aneurysm borders and proceeded, slice by slice, taking into account the previous hypothesis. The complete
3D model allowed us to understand whether the segmentation hypothesis for each slice (or series of a few slices with use of the 3D pen) was correct. The surgeon, seeing the 3D model (Fig. 6, right), decided for an endovascular stent instead of open surgery.

Case 8: robotic treatment for an aneurysm of splenic artery 1

In this case, manual refining with the touch screen was applied just to elongate small arterial branches not fully recognizable using the semiautomatic algorithm. The 3D reconstruction allowed a clear understanding concerning the precise position of the aneurysm's afferent and efferent arteries and their relations with the splenic vein and the pancreatic tail (Fig. 7A). This is an important aspect of this type of intervention. After visualization of the relationship between the various anatomic structures, the surgeon 


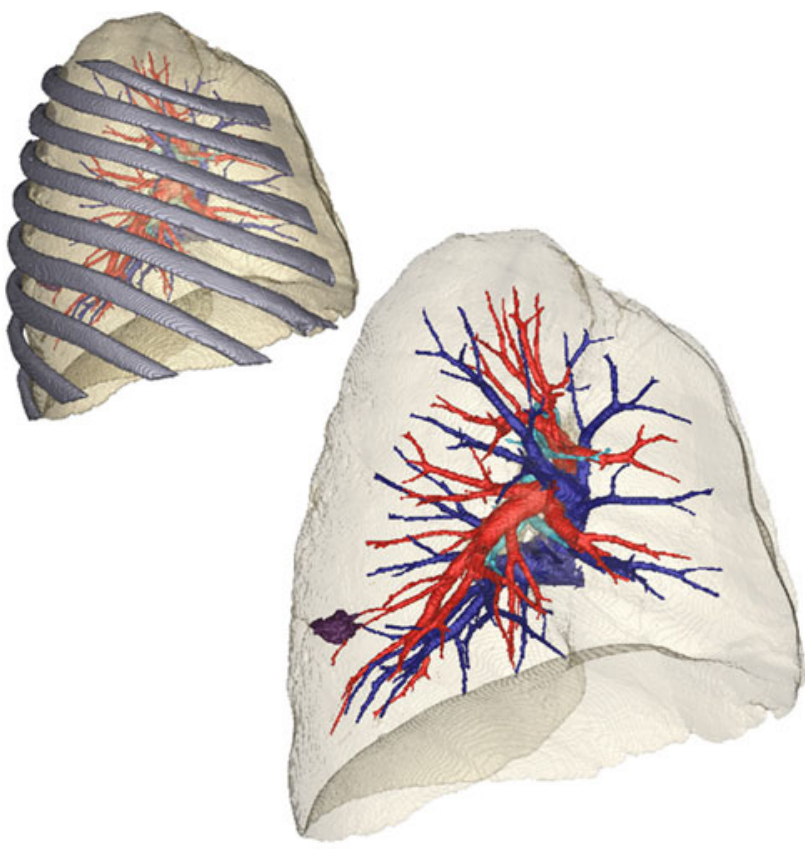

Fig. 5 Three-dimensional (3D) model of the lung with and without the rib cage. The position of the ribs is important for planning the insertion points for the trocars. On the other hand, the transparent image facilitates understanding concerning the position of the tumor and its relationship with the hilar structures

decided to proceed with a laparoscopic approach using the robot.

Case 9: robotic treatment for an aneurysm of splenic artery 2

Also in this case, starting with the rough model obtained using the semiautomatic algorithm, we manually refined the aneurysm morphology using the touch screen. As mentioned earlier, this type of reconstruction allows a clear understanding concerning the precise position of the aneurysm's afferent and efferent arteries and their relations to the pancreatic tail (Fig. 7D). This information was thought necessary to determine the feasibility of a robotic laparoscopic approach to the condition and to plan it [17]. Note that the standard CT images (Fig. 7C) and the direct volume rendering do not allow understanding that the aneurysm involves main bifurcation of the splenic artery close to the parenchyma and that the case involves one afferent and two efferent arteries. Low enhancement of the CT images required, after an initial automatic segmentation, manual refinement to delineate the topology and morphology of the vessels as in case 8 . In addition, the 3D model showed that the lower pole of the spleen also was supplied by a small collateral artery. After intraoperative exclusion of the aneurysm, thanks to this small collateral artery, the surgeon performed only one anastomosis, between the afferent artery and the efferent artery directed to the upper pole, avoiding a second anastomosis, because the lower pole was supplied by it.

\section{Case 10: megaspleen}

The patient in this case had a megaspleen that deformed the abdomen and stirred internal organs and vessels, which were twisted and kinked. The obtained 3D model (Fig. 8) offered the surgeon a better understanding of the deformed anatomy. A splenectomy was planned and performed through a hand-assisted laparoscopic approach. Our software also was used to predict in advance the volume of the spleen $\left(5,976 \mathrm{~cm}^{3}\right)$, showing high precision. In this case, the topology of the anatomy was not as complex as for other
Fig. 6 Left direct volume rendering of an aneurysm close to the bifurcation of the common hepatic artery involving the proper hepatic artery and the gastroduodenal artery. This image does not permit an understanding of its origin. Right three-dimensional (3D) model obtained through segmentation, which shows exactly that the aneurysm involves only the common hepatic artery. In the background, one of the computed tomography (CT) slices can be seen with the aneurysm highlighted in the red circle

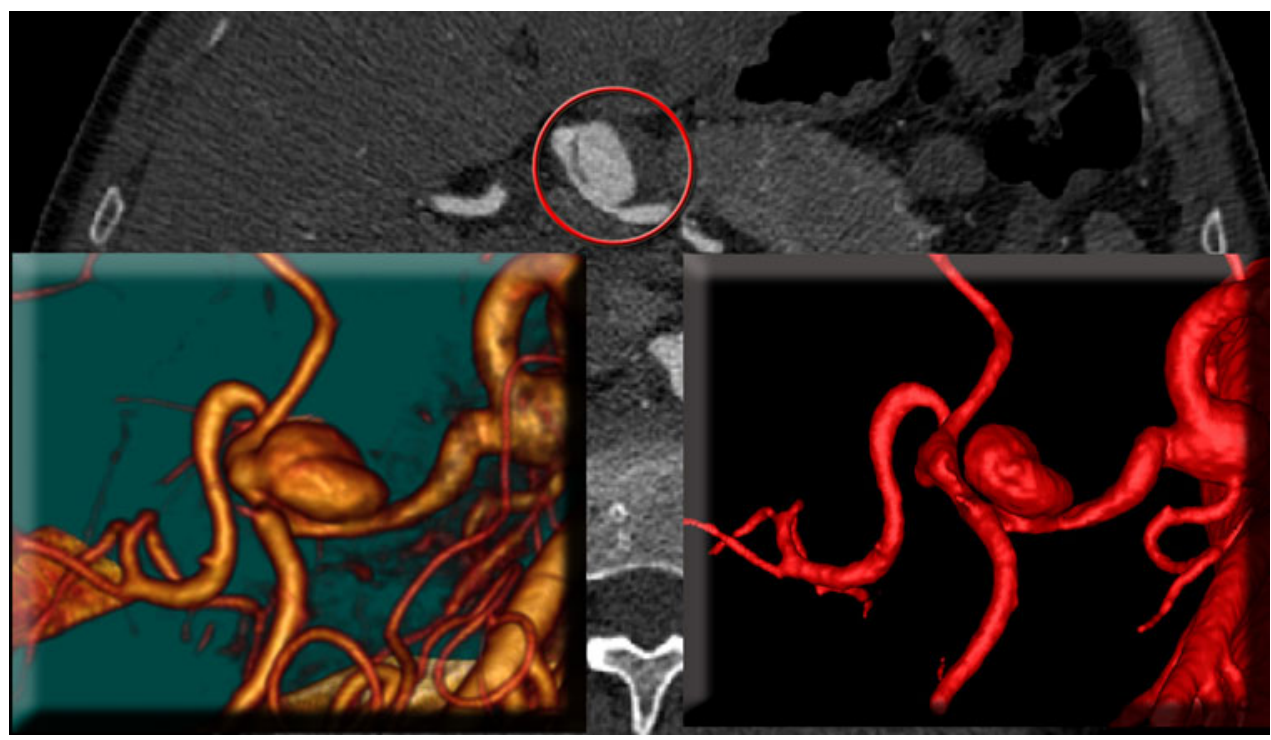



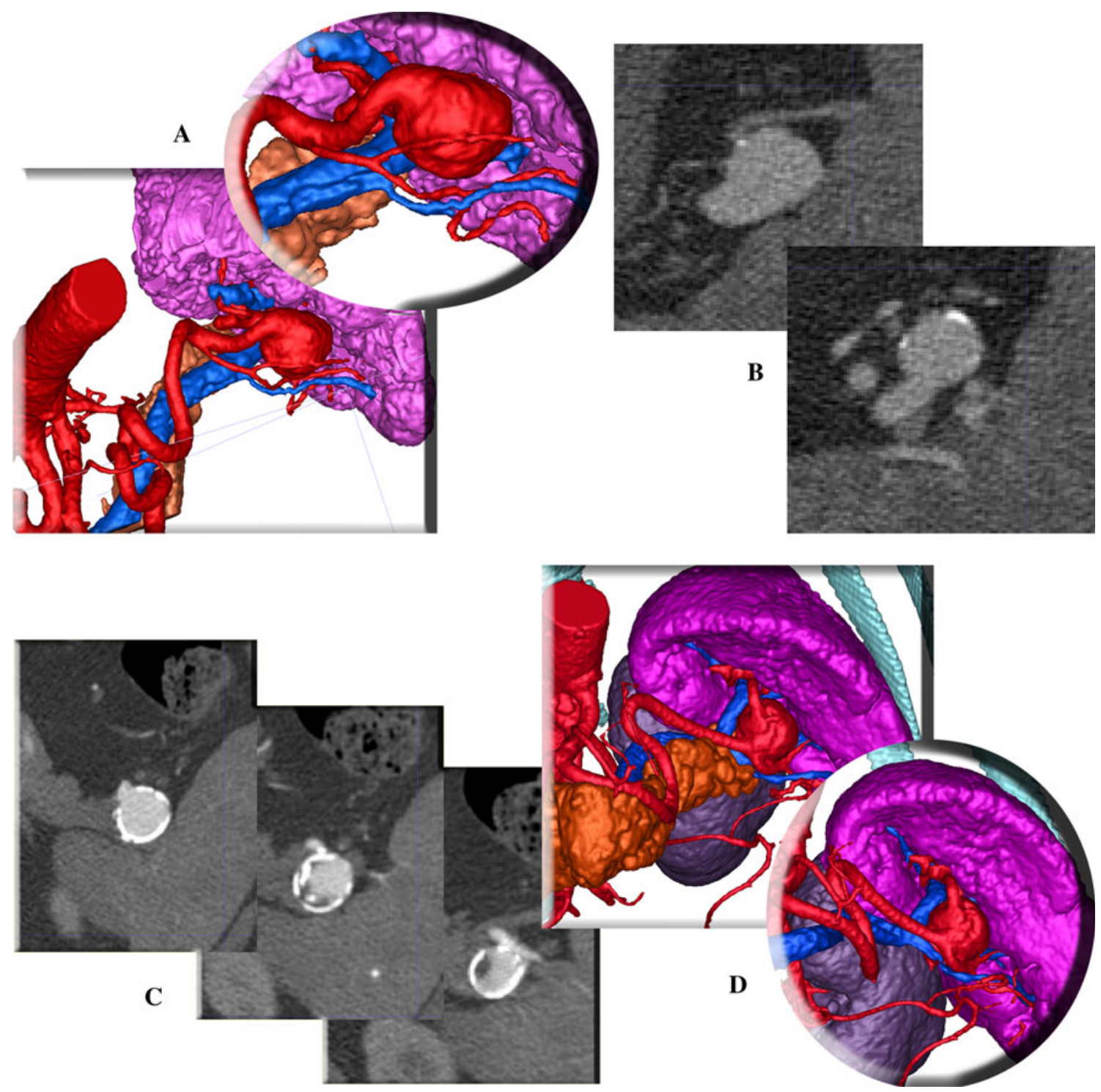

Fig. 7 Two cases of splenic artery aneurysm. (A, B), the threedimensional (3D) model, and the corresponding computed tomography (CT) slices for case 8. C The CT slices for case 9 show the

types of interventions, so the value of the segmentation for planning and executing this intervention was marginal.

Table 1 presents the surgeon's opinion for each case.

\section{Discussion}

This report describes the advantages offered by patientspecific 3D models generated segmenting MDCT images for the planning of general surgery interventions and provides some practical examples.

Preoperative planning in general surgery often is based on images acquired using CT. New-generation MDCT scanners are potentially capable of providing accurate ambiguity of afferent and efferent vessels. D The corresponding 3D model with and without the pancreatic tail facilitates understanding of the vascular anatomy and its anatomic relationship

information on the surgical anatomy. However, part of this information usually is lost through the current method of radiologic reporting or not fully used. Three-dimensional reconstructions are best generated by radiologists with the aid of surgeons working side by side. This type of collaboration is the key to producing reliable 3D models for preoperative planning and to guiding the proposed intervention. For this reason, our team involves three professional figures: radiologists (who know how to interpret radiologic images correctly), engineers for the development of new tools and sometimes for their usage (in close collaboration with the doctor), and surgeons (who know the anatomic structures involved in each type of intervention). 
Fig. 8 Three-dimensional (3D) model of a megaspleen. It is possible precisely to understand the topology of vessels and to delineate the deformed pancreatic tail

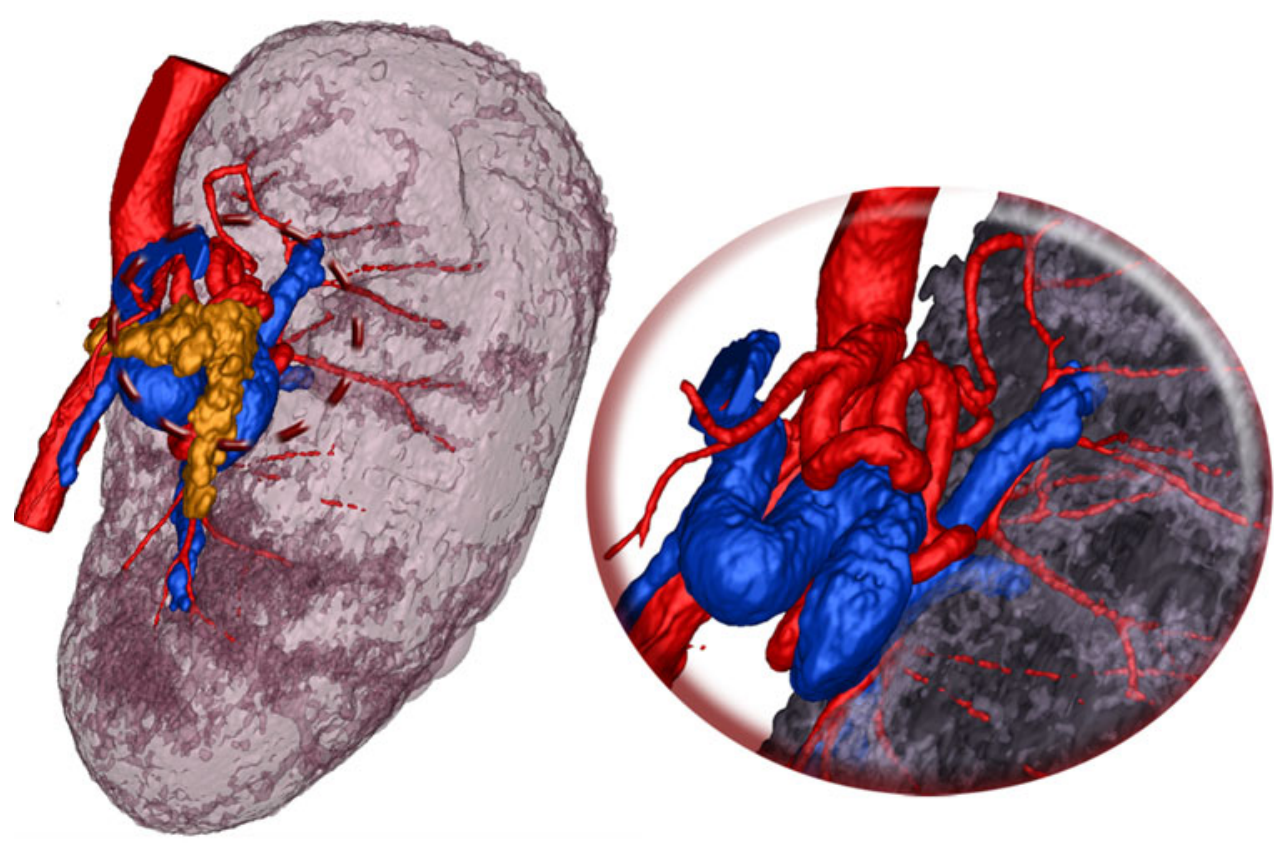

On the basis of our experience, modern MIP or direct volume-rendering visualization tools, which offer highquality results for visualization of CT angiographic images, sometimes do not allow full exploitation of the information contained in the radiologic data set. In these cases, the segmentation of MDCT images can be a useful instrument for planning critical surgical interventions.

Currently, the market and the open source community offer automatic, semiautomatic, and manual segmentation tools sometimes integrated into the radiologic software. Regarding automatic and semiautomatic tools, the need to validate the result must be taken into account. A wrongly segmented image can cause terrible results in terms of surgical planning and outcome. The segmented data set must be validated by a radiologist or by an expert surgeon before its usage. Validation done directly at the workstation during the segmentation work must be preferred over postvalidation work because the user who sees the growing of each segmented anatomy, one by one, can pay implicitly more attention to the global result than the radiologist who must validate a segmentation performed previously, sliding entirely on a "colored" volume superimposed on gray scale images (the traditional validating method for segmentation).

Regarding manual segmentation, necessary for difficult images due to difficult anatomic structures or poorly acquired images, the possibility of using a touch screen monitor to speed up the work should be considered. When the CT images are difficult to interpret, a proven winning strategy is to try starting with the slices whose anatomic borders are more clear and then to proceed, slice by slice, taking into account any previous hypothesis. The complete
3D model allows understanding of whether the segmentation hypothesis, applied for each slice, is right. This approach was particularly useful for cases 7, 8, and 9 .

For difficult cases, segmentation in fact allows better understanding of medical images, reducing the mental stress of keeping in mind all the mental passages and consequently improving the radiologist's potentialities. Manual segmentation sometimes allows a better understanding of medical images, avoiding doubts concerning artery topology raised by the use of direct volume-rendering visualization.

Analysis of our preliminary data confirmed that this type of image reconstruction can be of great value in many different settings for treatment planning and for teaching purposes. Overall mean values are inserted in the table to indicate the utility of the segmentation for the different domains (areas and types of interventions). Even if from their analysis a global result for each domain cannot be extrapolated because the number of trials is not statistically sufficient for extrapolating the probability of correlated variables (areas vs types of intervention), they can be useful for performing some considerations.

Clearly, a 3D representation for "visualization of the target anatomy" is intrinsically more easy and useful than a simple 2D visualization. In fact, this point received the maximum score. Nonetheless, 3D reconstruction remains marginally used in clinical practice. This high score (5) encourages the use of $3 \mathrm{D}$ reconstruction to improve visualization of the target.

Segmentation allows a better description of the anatomy involved in the intervention for the surgical staff, students, and resident surgeons, particularly in the case of lesions in 
complex anatomies such as those of the liver or the lung. These anatomies are difficult to describe using only a blackboard because they require illustration of multiple anatomic structures with complex 3D developments close to each other.

Segmentation allows a better understanding concerning the anatomic relationship of the target structures. This in turn brought a change in the surgical strategy for 6 of 10 cases in the current series (scores 4 and $5=$ good and very good). Volumetric analysis also was helpful in two cases (case 2 and 3) of proposed major liver surgery, providing a reliable assessment of liver regeneration after segmental portal vein embolization.

In the operating room, the patient-specific 3D model obtained after segmentation allows the intervention to proceed with the knowledge of exactly which vessels to cut and which anastomosis to execute. The time required for searching every vessel to be excluded can be reduced, and the surgeon can approach critical interventions with a major awareness of the patient-specific anatomy. In any case, the value offered by the segmentation depends on the complexity of the anatomy involved in the intervention. In fact, splenectomy (case 10) and lung lobectomy (case 6) in the current series received a lower mean score than other types of interventions.

Intraoperative augmented reality and navigation also can be based on the generation of MDTC 3D models. It was used for two cases in our series (neuroendocrine pancreatic tumor [case 5] and splenic artery aneurysm [case 9]), showing that the technique can be adopted also for abdominal surgery, although with some limitation. In fact, both the body of the pancreas and the splenic artery are relatively fixed structures, which only minimally modify their anatomic position within the abdomen as the patient is moved and the decubitus is changed. For the majority of the most common abdominal surgical procedures, organ shifting and tissue deformation during the operation remain unsolved problems that restrict image-guided navigation to a few anatomic areas.

Although the generation of 3D models segmenting images, using our software or others, remains a complex procedure that is considerably time consuming, its value, as evidenced by this preliminary report, is highly recognized by the final user, the operating surgeon. However, it also can be perceived by nonmedical staff according to the results for cases in which the segmentation allowed the choice of a better surgical strategy (i.e., endovascular instead of open surgery as in case 7). Further study is necessary to quantify the improvements of the segmentation for each type of complicated intervention in terms of operative blood loss, duration of intervention, morbidity and mortality, and hospital stay.
Acknowledgments This work was supported by Fondazione Cassa di Risparmio di Pisa (project 103-07).

Disclosures Vincenzo Ferrari, Marina Carbone, Carla Cappelli, Luigi Boni, Franca Melfi, Mauro Ferrari, Franco Mosca, and Andrea Pietrabissa have no conflicts of interest or financial ties to disclose.

Open Access This article is distributed under the terms of the Creative Commons Attribution Noncommercial License which permits any noncommercial use, distribution, and reproduction in any medium, provided the original author(s) and source are credited.

\section{References}

1. Dawes TJ, Vowler SL, Allen CM, Dixon AK (2004) Training improves medical student performance in image interpretation. Br J Radiol 77:775-776

2. Fishman EK, Ney DR, Heath DG, Corl FM, Horton KM, Johnson PT (2006) Volume rendering versus maximum intensity projection in CT angiography: what works best, when, and why. Radiographics 26:905-922

3. Ishikawa T, UshikI T, Mizuno K, Togashi T, Watanabe K, Seki K, Ohta H, Yoshida T, Takeda K, Kamimura T (2005) CT-maximum intensity projection is a clinically useful modality for the detection of gastric varices. World J Gastroenterol 11:7515-7519

4. Mori G, Tokunaga D, Takahashi KA, Hojo T, Fujiwara H, Arai Y, Taniguchi D, Takatori R, Imai K, Otakara E, Ito H, Nishimura $\mathrm{T}$, Kubo T (2008) Maximum intensity projection as a tool to diagnose early rheumatoid arthritis. Mod Rheumatol 18:247-251

5. Valencia R, Denecke T, Lehmkuhl L, Fischbach F, Felix R, Knollmann F (2006) Value of axial and coronal maximum intensity projection (MIP) images in the detection of pulmonary nodules by multislice spiral CT: comparison with axial 1-mm and 5-mm slices. Eur Radiol 16:325-332

6. Kamai T, Furuya N, Kambara T, Abe H, Honda M, Shioyama Y, Kaji Y, Yoshida K (2010) Single minimum incision endoscopic radical nephrectomy for renal tumors with preoperative virtual navigation using 3D-CT volume rendering. BMC Urol 10:7

7. Coll DM, Uzzo RG, Herts BR, Davros WJ, Wirth SL, Novick AC (1999) 3-dimensional volume rendered computerized tomography for preoperative evaluation and intraoperative treatment of patients undergoing nephron-sparing surgery. J Urol 161:1097-1102

8. D'Andrilli A, Vismara L, Rolla M, Ibrahim M, Venuta F, Pochesci I, Masciangelo R, Rendina EA (2009) Computed tomography with volume rendering for the evaluation of parenchymal hyperinflation after bronchoscopic lung volume reduction. Eur J Cardiothorac Surg 35:403-407

9. Peters TM (2006) Image guidance for surgical procedures. Phys Med Biol 51:R505-R540

10. Peters TM (2000) Image-guided surgery: from $\mathrm{x}$-rays to virtual reality. Comput Methods Biomech Biomed Eng 4:27-57

11. Lamata P, Lamata F, Sojar V, Makowski P, Massoptier L, Casciaro S, Ali W, Studeli T, Declerck J, Elle OJ, Edwin B (2010) Use of the Resection Map system as guidance during hepatectomy. Surg Endosc 24:2327-2337

12. Roguin A, Beyar R (2010) Real case virtual reality training prior to carotid artery stenting. Catheter Cardiovasc Interv 75:279-282

13. Hislop SJ, Hedrick JH, Singh MJ, Rhodes JM, Gillespie DL, Johansson M, Illig KA (2009) Simulation case rehearsals for carotid artery stenting. Eur J Vasc Endovasc Surg 38:750-754

14. Ferrari V, Cappelli C, Megali G, Pietrabissa A (2008) An anatomydriven approach for generation of 3D models from multi-phase CT images. Int J Comput Assist Radiol Surg 3 (Supp 1):271-273 
15. Yushkevich PA, Piven J, Hazlett HC, Smith RG, Ho S, Gee JC, Gerig G (2006) User-guided 3D active contour segmentation of anatomical structures: significantly improved efficiency and reliability. Neuroimage 31:1116-1128

16. Megali G, Ferrari V, Freschi C, Morabito B, Cavallo F, Turini G, Troia E, Cappelli C, Pietrabissa A, Tonet O, Cuschieri A, Dario P, Mosca F (2008) EndoCAS navigator platform: a common platform for computer and robotic assistance in minimally invasive surgery. Int J Med Robot Comput Assist Surg 4:242-251

17. Pietrabissa A, Morelli L, Ferrari M, Peri A, Ferrari V, Moglia A, Pugliese L, Guarracino F, Mosca F (2010) Mixed reality for robotic treatment of a splenic artery aneurysm. Surg Endosc 24:1204 